\title{
Histology of the Suprapubic and Anogenital Cutaneous Glands in Male Cotton Top Tamarins (Saguinus oedipus)
}

\author{
Sara Fontani ${ }^{a} \quad$ Gianfranco Tanteri $^{\mathrm{b}}$ Stefano Vaglio ${ }^{\mathrm{a}, \mathrm{c}}$ \\ Giovanni Delfino $^{b}$ Jacopo Moggi-Cecchi ${ }^{a}$ \\ Laboratories of a Anthropology and ${ }^{\mathrm{b} C}$ Comparative Anatomy, Department of Biology, \\ University of Florence, Florence, Italy; ' ${ }^{\mathrm{C}}$ Department of Anthropology and Behaviour, \\ Ecology and Evolution Research Centre, Durham University, Durham, UK
}

\section{Key Words}

Cotton top tamarin · Histology · Light microscope · Saguinus oedipus · Scent glands $\cdot$ Scent marking

\begin{abstract}
In cotton top tamarins (Saguinus oedipus), scent glands have been mostly studied in females from museum collections. This work aims to extend the investigation to male specimens, introducing a novel source of skin samples. Two adult males from zoo populations, one intact and one castrated, were immediately frozen after natural death. Skin samples were later collected at the thawing onset, soaked with cold fixative and processed for light microscopy. Sebaceous units of scent glands showed phasic secretory activity in the intact male and marked fibrosis in the castrated male. It appears, therefore, that light microscopy samples from frozen tissues provide detailed features that can disclose distinctive traits in specimens characterized by different hormonal balances.
\end{abstract}

(c) 2014 S. Karger AG, Basel

\section{Introduction}

Chemical communication plays fundamental roles in several animal taxa [Alaux et al., 2010]. Scent molecules are mostly volatile and convey information to individuals of the same or different species [Albone and Shirley, 1984; Setchell et

\begin{tabular}{ll}
\hline KARGER & $\oplus 2014$ S. Karger AG, Basel \\
E-Mail karger@karger.com & $0015-5713 / 14 / 0852-0109 \$ 39.50 / 0$ \\
www.karger.com/fpr
\end{tabular}

Prof. Giovanni Delfino

Department of Biology, University of Florence

Via La Pira 4

IT-50121 Florence (Italy)

E-Mail giovanni.delfino@unifi.it 
al., 2010, 2011]. In most vertebrates, such compounds are released from complex cutaneous gland bodies [French and Snowdon, 1981] in association with particular behaviours.

Confirming the adaptive flexibility of secretory cell lines in the skin of vertebrates, scent glands are composed of constitutive cutaneous exocrine units that form conspicuous gland bodies in specific areas [Quagliata et al., 2006]. In mammals, scent glands are associated with hair follicles and include specialized sebaceous (holocrine and acinar) glands (SGs) and apocrine (tubular) glands (AGs). Both are derived from a common hair follicle anlage and release their products onto the body surface through the hair channel with the aid of the hair erector muscles [Ozaki et al., 2004]. AGs produce the appropriate scent molecules, whereas SGs manufacture a carrier that delays degradation of scent molecules after release [Welsch et al., 1998; Stoeckelhuber et al., 2000].

Strepsirhine primates use olfactory cues to communicate various information, such as to assess a signaller's reproductive state, dominance status or individual identity, and to modulate the reproductive competence of conspecifics [Epple et al., 1980; reviewed in Scordato and Drea, 2007]. The cotton top tamarin (Saguinus oedipus) exhibits two patterns of scent-marking behaviour that involve the anogenital and suprapubic regions and are more frequent in females than males [French and Snowdon, 1981]. Morphological studies from the family Callitrichidae reveal thick clusters of SGs and AGs in the anogenital and suprapubic skin that differ between species [French and Cleveland, 1984] and are more developed in females than in males [Perkins, 1969].

The aims of this study, employing light microscopy, are: (1) to provide an alternative approach to the traditional use of animals from museum collections (that have undergone a prolonged in toto fixation with formic aldehyde [Moraes et al., 2006]) by investigating specimens frozen immediately after natural death; (2) to further the understanding of male glands that, to date, have been largely neglected.

\section{Methods}

In order to get specimens suitable for investigations, we founded a collaborative network between the University of Florence and Italian zoological gardens. Skin fragments were collected from 2 adult male tamarins that had been frozen after their natural deaths. The animals had been housed at the Pistoia Zoological Garden and the Punta Verde Zoological Park, respectively (both in Italy). The tamarin at the Pistoia Zoological Garden had undergone castration in accordance with the European Endangered Species Breeding Programs protocol.

During defrosting, the veterinary surgeon removed skin samples $\left(25 \mathrm{~mm}^{2}\right.$ of free surface, and thickness ranging from 5 to $7 \mathrm{~mm}$ ) from 7 cutaneous areas: 3 in the suprapubic (proximal, intermediate and distal to-from the external genital organs) and 4 in the perianal (cephalic, left and right lateral, and caudal) regions. Immediately after removal, samples were soaked in a $4{ }^{\circ} \mathrm{C}$ solution of: saturated aqueous picric acid $(15 \mathrm{ml})$, formic aldehyde $(5 \mathrm{ml})$ and glacial acetic acid $(1 \mathrm{ml})$; this was kept at room temperature. This deeply penetrating solution, Bouin's fluid, is suitable for speedy, progressive fixation of large tissue samples [Mazzi, 1977]. Fixed samples were processed for ordinary light microscopy in the Comparative Anatomy Laboratory of the Department of Biology (University of Florence). After $24 \mathrm{~h}$, samples were rinsed in 50\% ethanol to remove excess picric acid, dehydrated with increasing ethanol series, cleared with xylene and embedded in paraffin according to routine methods. A total of approximately $4.8 \times 1,000$ serial $7-\mu \mathrm{m}$-thick sections were obtained with a rotary microtome (Dialux $20 \mathrm{~EB}$; Leitz, Wetzlar, Germany), to be stained with haematoxylin and eosin (850 sections, for the main investigation) or 
Mallory's trichrome (320 sections, to obtain clear-cut images). Micrographs were collected with a digital camera (Coolpix 4500; Nikon, Tokyo, Japan) connected to a microscope (model 1512; Leitz) and processed with Adobe Photoshop CS2 (Adobe Systems Inc., version 9.0.2) to obtain compound images with homogeneous contrast of grey tones. This was achieved using image adjustment tools in this sequence: curves, levels and brightness/contrast.

\section{Results}

In both specimens, the investigated cutaneous areas appeared largely hairless (fig. 1a, b, 2a, b, 3b, 4a, b). Hairs were of the vellus type, with thin cortex and reduced pigmentation (fig. $3 c, d, 4 c, d$ ). Vellus follicles showed the usual pattern of concentric cell layers in transverse sections (fig. 1f, 2c) and were associated with AGs and SGs. Follicles associated with SGs formed the pilosebaceous units. AGs appeared as coiled, secretory bulks, with a thick arrangement of tubules in deep dermal layers (fig. 1a, b, 2a, b, 4a). AGs had straight ducts with stratified walls and exhibited branched organization in intermediate dermal layers (fig. 1e). SGs were large in size and displayed specialized features according to the topographical criteria stated above.

\section{Suprapubic Areas}

The suprapubic areas of the intact male contained SGs consisting of several secretory units, either acinar or short-tubular in shape (fig. 1a), which drained into a main duct merging into the follicle through a dilated tract (fig. 1a, c). In transverse sections, the secretory units had a flower-like arrangement due to their radial convergence toward the central follicle (fig. 1c, f). In corresponding areas, SGs of the castrated animal showed ducts protruding from dense connective tissue sheaths around the secretory units (fig. 1b). Sebaceous units resembled solid tubules converging towards the duct (fig. 1d) that consisted of a sinuous tract (fig. 1e) and a somewhat enlarged terminal portion (fig. 1d, e). These secretory units, of intermediate density, alternated with thick septa that were continuous with the gland sheath (fig. 1d). In both specimens, sebaceous cells exhibited holocrine processes directed toward the duct (fig. 1c, d, f) and gave rise to products with comparable features (fig. 1a, c-e).

\section{Cephalic Perianal Area}

SGs with the traits described above were abundant in the cephalic perianal area of both specimens (fig. $2 \mathrm{a}, \mathrm{b}$ ). In the intact male, secretory units were acinar, with an intermediate cytoplasmic density that decreased toward the light compartment of the dilated collecting duct (fig. 2c). Thin connective-tissue laminae separated the sebaceous acini and followed their radial arrangement (fig. 2c). SGs of the castrated specimen ranged from ellipsoid to pear-shaped, with the largest axis orthogonal to the skin surface (fig. 2b, d). These solid and dense bodies included secretory units and ducts (fig. 2b). They largely consisted of a connective-tissue sheath that was denser than the ordinary glandular stroma (fig. 2b). The stroma gave rise to thin screens that were reinforced by additional layers of connective tissue and formed thick septa between secretory units (fig. 2d). These were club-like acini, with diameters comparable to the width of the initial tract of their duct (fig. $2 \mathrm{~d}, \mathrm{e}$ ), which continued along a coiled 

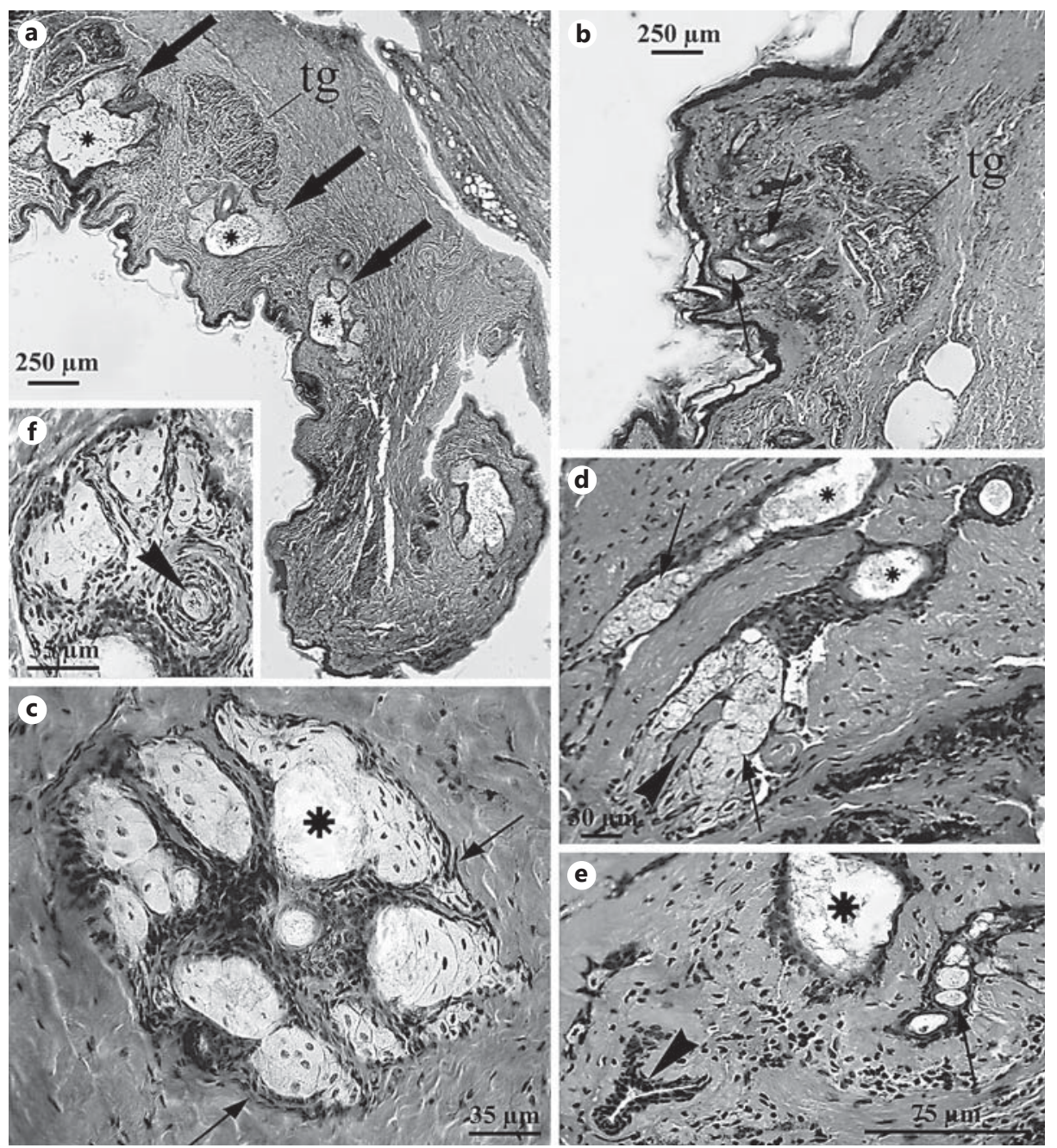

Fig. 1. Suprapubic areas from the intact male $(\mathbf{a}, \mathbf{c}, \mathbf{f})$ and the castrated male $(\mathbf{b}, \mathbf{d}, \mathbf{e})$. Haematoxylin-eosin staining is shown in $\mathbf{a}, \mathbf{b}, \mathbf{d}$ and $\mathbf{e}$; Mallory's trichrome staining is shown in $\mathbf{C}$ and $\mathbf{f}$. $\operatorname{tg}=$ Apocrine, tubular glands (AGs). a Large, specialized SGs occupy the dermal layers. Acini (arrows) and ducts appear with enlarged and semitransparent lumens (asterisks). AGs appear as thick clusters of coiled secretory units in the deep dermal layers. b SGs are recognizable by ducts with content of intermediate density (arrows). A cluster of AGs is also present in the image. c Sebaceous acini consisting of lipogenic cells organized in a radial arrangement. The holocrine process is emphasized when comparing the flat and dense basal cells (arrows) with the light secretory mass in the distal acinar tracts and duct (asterisk). d Contiguous sebaceous acini resembling solid chords of secretory cells (arrows) involved in holocrine processes. The ducts (asterisks) contain dispersed materials, and a septum of connective tissue (arrowhead) protrudes toward the sebaceous units. e A sebaceous duct displaying sinuous (arrow) and enlarged (asterisk) tracts. The arrowhead points to the branched duct of the AG duct accompanying the SG duct. f A pilosebaceous unit with acini and a villus hair follicle (arrowhead). 

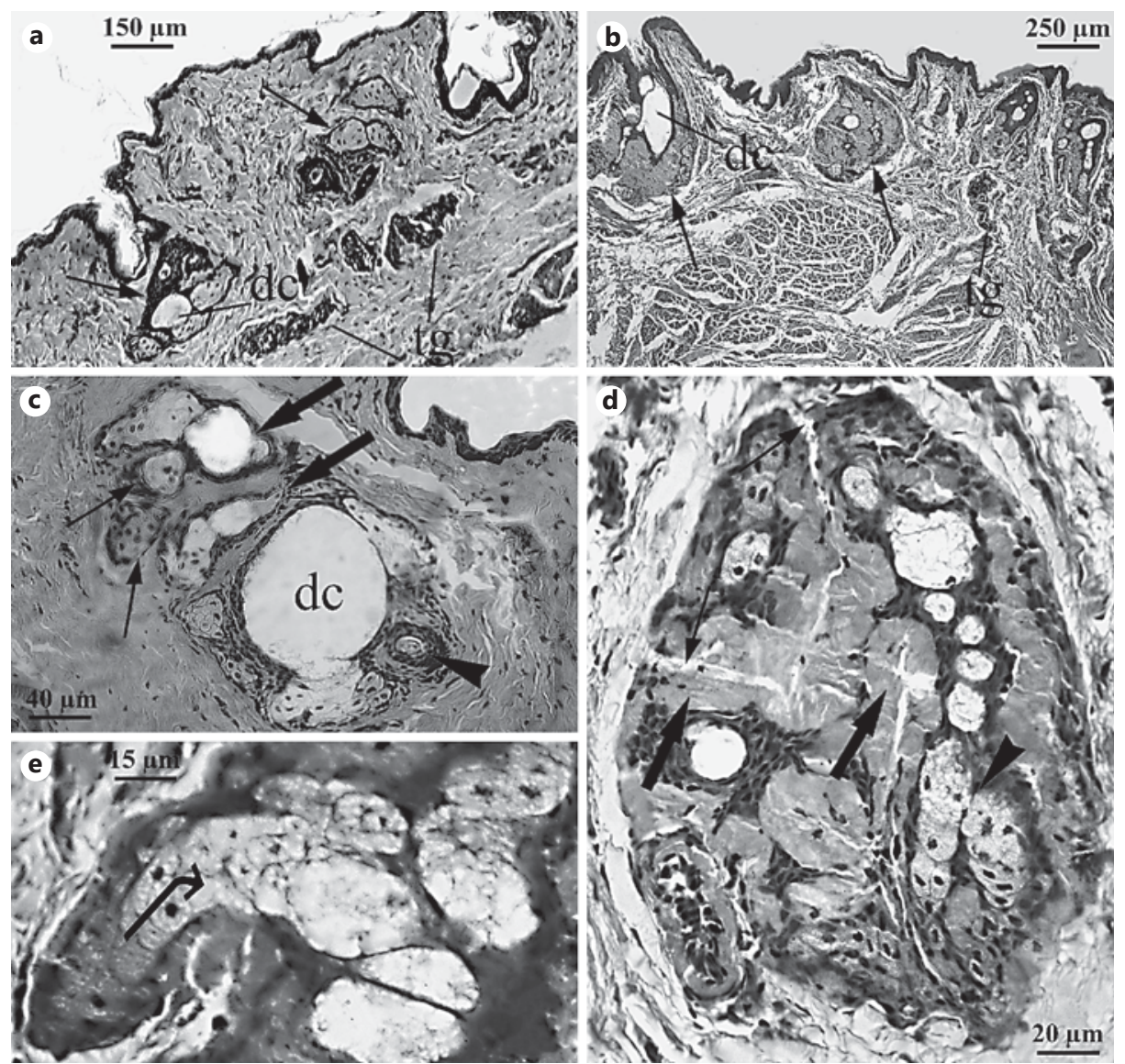

Fig. 2. Scent glands from the cephalic end of the anogenital area (antero-anal region) of the intact male $(\mathbf{a}, \mathbf{c})$ and the castrated male $(\mathbf{b}, \mathbf{d}, \mathbf{e})$. Haematoxylin-eosin staining is shown in $\mathbf{b}, \mathbf{d}$ and $\mathbf{e}$; Mallory's trichrome staining is shown in a and $\mathbf{c}$. dc = Duct of SG; $\operatorname{tg}=$ apocrine, tubular glands (AGs). a Specialized SGs at low magnification. Ducts are dilated and contain a transparent product. Acini are enlarged, and secretory cells show homogeneous cytoplasm density (arrows). AGs appear beneath the sebaceous acini. b SGs appear as dense structures (arrows) and are recognizable by the light compartments of their ducts. AG glands occupy the deeper dermal layers. c Representative patterns of specialized SGs. To notice sequential tracts of the dilated duct (large arrows), hair follicle (arrowhead) and acini arranged in a somewhat radial fashion (thin arrows), they are separated from each other by septa composed of moderately dense connective tissue. d A specialized SG displaying elongated secretory units (arrowhead) flanked by thick septa of connective tissue. Each septum consists of a thin inner layer that is continuous with the periglandular stroma (thin arrows) and a thick outer layer of denser connective tissue (large arrows). e A sebaceous unit composed of secretory cells with decreasing cytoplasmic density gradients (bowed arrow). 
course into the dilated terminal tract (fig. 2d). In both specimens, secretory cells (adenocytes) underwent holocrine disintegration along the acinus axis that appeared as a follicle-centred, foamy degeneration and was marked by cytoplasmic accumulation of lipid droplets. Additionally, pyknosis and karyorrhexis (i.e. nuclear shrinkage and fragmentation) were noted (fig. 2c-e).
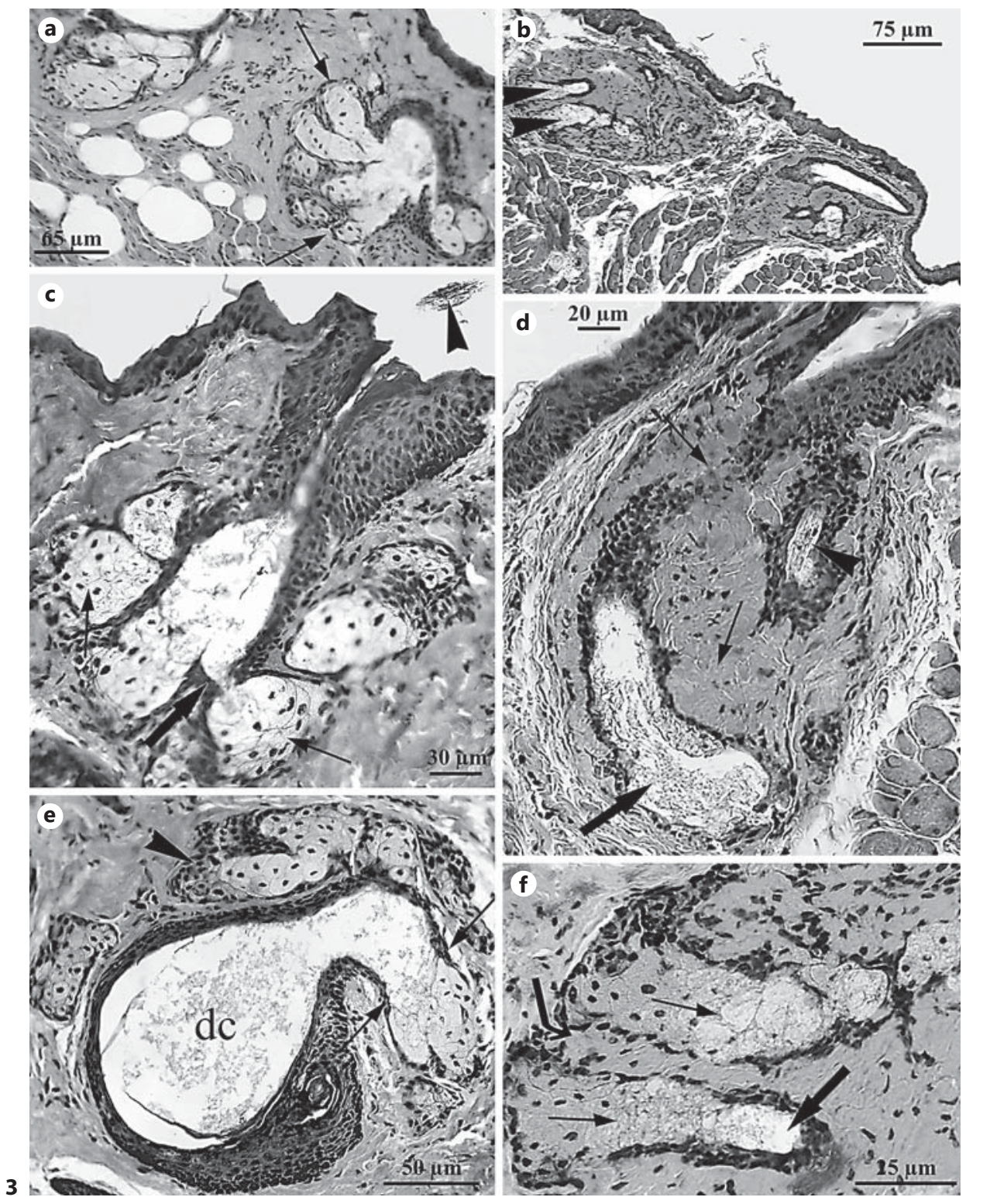

(For legend see next page.)

$114 \quad$ Folia Primatol 2014;85:109-118 DOI: $10.1159 / 000360221$

Fontani/Tanteri/Vaglio/Delfino/Moggi-Cecchi 


\section{Caudal Perianal Area}

SGs in samples from the caudal perianal area of both specimens confirmed previous findings. SGs in the intact male were radially arranged acini (fig. 3a) converging toward dilated ducts, and their widths contrasted markedly with the small compartments of the vellus hair channels (fig. 3c). An intermediate density of sebaceous cells arranged in several layers was observed between the light sebum inside the collecting duct and the opaque basal cells (fig. 3c). These undifferentiated adenocytes (adenoblasts) had remarkably high nucleoplasmic ratios compared to partially or fully differentiated sebaceous cells (fig. 3c). Some secretory units had terminal portions resembling proper acinar ducts that independently drained into the common collecting duct and, thus, indicated a compound glandular structure (fig. 3c, e). The common duct had a wide lumen that stored remarkable amounts of sebum, suggesting it is not merely a draining tract (fig. 3e). In the castrated specimen, SGs consisted of secretory units embedded in dense connective tissue (fig. 3b, f). The thick connective tissue sheath largely obscured the arrangement of the secretory units (fig. 3d), which alternated with inner septa of connective tissue in longitudinal sections (fig. 3b, f) and displayed the typical gradient of holocrine degeneration toward the duct (fig. 3f).

\section{Lateral Perianal Areas}

The paired cutaneous areas flanking the anogenital axes of both specimens contained a few ordinary SGs that were relatively branched in the intact male (fig. 4a). In the castrated specimen, SGs consisted of a few small secretory units; therefore, lowpower magnification showed pilosebaceous units primarily consisting of vellus hair follicles (fig. 4b). High-power magnification in both specimens revealed structural patterns consistent with ordinary SGs (fig. 4c, d), including lack of proper ducts and dilated specialized tracts. Groups of coiled tubules consistent with AGs were found in the deeper dermal layers (fig. 4a).

\section{Discussion and Conclusion}

Present findings demonstrate that frozen specimens provide cutaneous samples suitable for ordinary light microscope analysis. Noticeably, images depicting patterns of progressive, polarized holocriny in sebaceous acini are more informative than features collected from museum specimens. Skin samples collected from these animals

Fig. 3. Specialized SGs from the postero-anal region in the intact male $(\mathbf{a}, \mathbf{c}, \mathbf{e})$ and the castrated male (b, d, f). Haematoxylin-eosin staining is shown in $\mathbf{b}-\mathbf{f} ;$ Mallory's trichrome staining is shown in $\mathbf{a}$. $\mathrm{dc}=$ Duct. $\mathbf{a}, \mathbf{b}$ Comparison of specialized SGs, in which the convergence angles of acini toward the duct are either wide (arrows) or narrow (arrowheads). c Structures of specialized SGs. The duct is dilated and continuous with the channel of the vellus hair (arrowhead). Thin arrows indicate secretory cells in intermediate stages of holocriny, while the large arrow indicates a proper acinar duct merging with the common SG duct. d SG in a semitangential section. A thick sheath of connective tissue (thin arrows) encompasses a dilated duct (large arrow) and hair channel (arrowhead). e Duct exhibiting a remarkably dilated lumen. Arrows indicate its junction with a short acinar duct, and the arrowhead indicates acinar stem cells. f Acini showing an obvious gradient of holocriny from the periphery (thin arrows) toward the duct (large arrow). The bowed arrow indicates the connective tissue sheath that is continuous with the inner septa. 

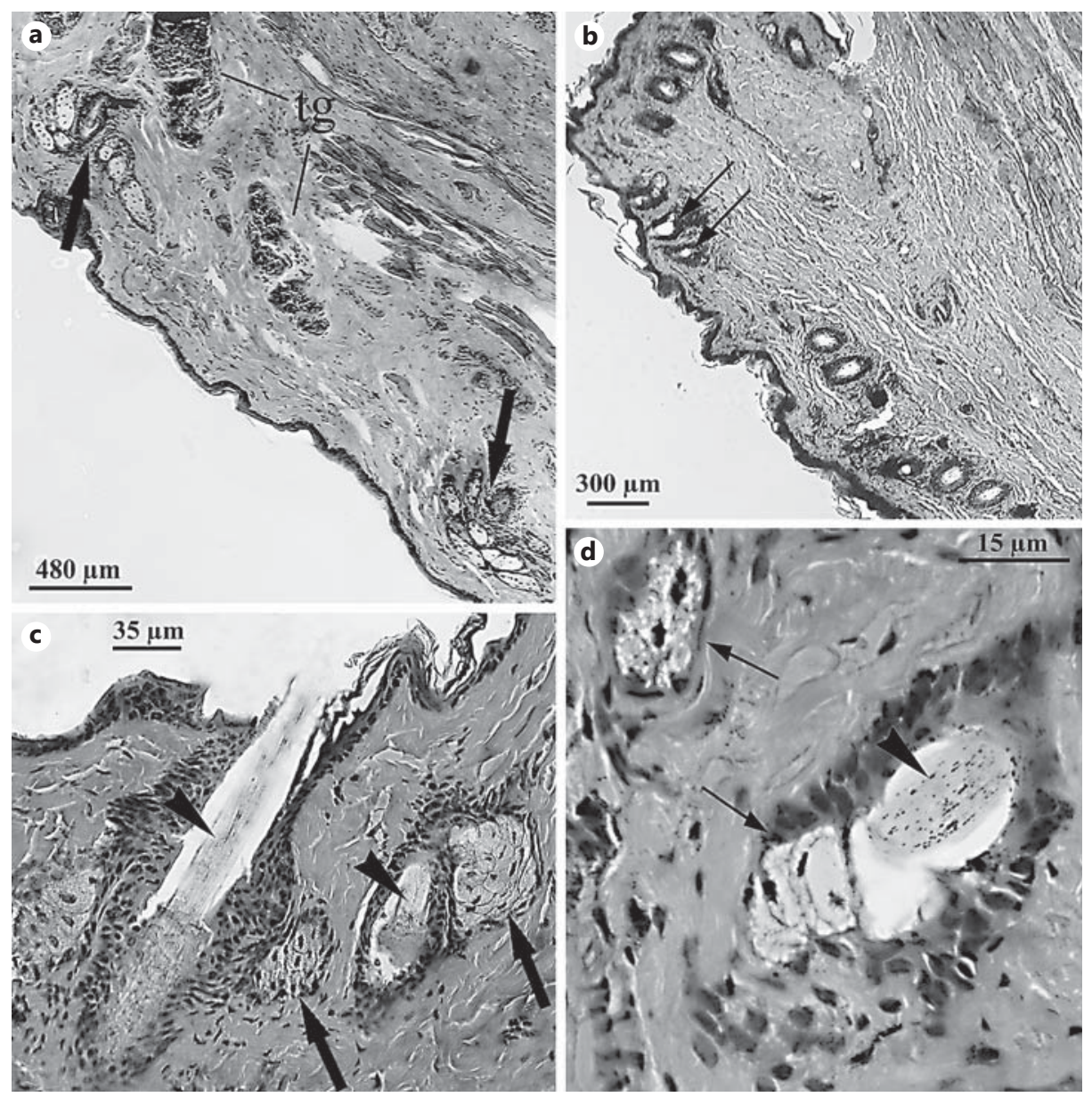

Fig. 4. Cutaneous areas from both lateral sides of the anogenital region. Specimens from the intact male $(\mathbf{a}, \mathbf{c})$ and the castrated male $(\mathbf{b}, \mathbf{d})$ are shown. Haematoxylin-eosin staining is shown in b-d; Mallory's trichrome staining is shown in a. $\operatorname{tg}=$ Apocrine, tubular glands (AGs). a Ordinary SGs consisting of a few elongated secretory units (arrows) converge toward the hair channels. AG clusters are observed in the deep dermis. b Hair channels (arrows) are associated with small SGs. The pilosebaceous units seem to lack any secretory components at low magnification. c Pilosebaceous units with medium-sized acini are indicated by arrows. Arrowheads indicate the vellus hairs. d An SG consisting of a few, small secretory units is indicated by arrows. The arrowhead indicates a vellus hair.

do allow cytological analysis [Moraes et al., 2006], but results provide a sharp differential step characterization of the holocrine process that is marked by an apoptotic feature gradient of lipogenic degeneration.

Moreover, the present data further our understanding of scent glands in male tamarins. AGs and SGs from suprapubic areas in males of the species S. oedipus represent rostral continuations of the specialized glands found in the unpaired anogeni- 
tal region, but they resemble the ordinary type in adjacent paired areas. A linear suprapubic-anogenital distribution of scent glands is consistent with the 'suprapubic marking' of tamarins [French and Snowdon, 1981; French and Cleveland, 1984] that occurs by active traction with an alternating or one-way dragging or sprawling of the ventral trunk surface onto a substrate (Fontani, pers. observations).

AGs in scent glands resembled the ordinary type, but SGs showed specialized features that were especially noticeable in the intact male. SGs were large but did not reach the 'gigantic' size described in females [Perkins, 1969]. Peculiar features of scent SGs in males included a radial arrangement of acini and formation of a distinctive dilated reservoir devoted to product storage. Glandular products are stored in exocrine glands that exhibit phasic secretory discharge; it possibly occurs in scent-marking behaviour when friction is produced between the ventral body surface and a substrate. In contrast, 'ordinary' sebum is released continuously (i.e. through tonic activity) and provides constant protection to the hairs. The phasic activity of specialized SGs is consistent with the presence of basal pools of stem cells that undergo an intermittent secretory differentiation induced by the discharge of material from the reservoir during scent-marking behaviour.

The castrated male showed fibrosis patterns in specialized SGs, characterized by consistent features of stromal tissue growth between and around sebaceous acini. Since hairs and annexed glands are targets of androgenic steroids [Albone and Shirley, 1984; Chen and Zouboulis, 2009], it seems that sexual hormones may control the stromal components of SGs. It should be stressed that we cannot infer any causative relationship between castration and SG modifications, because of the small number of specimens investigated and the lack of a record about the age at surgery. Nevertheless, the data collected from the specimens under comparison confirm that the technique employed is suitable for the differential histological analysis of samples with a distinctive hormonal background.

\section{Acknowledgements}

We are grateful to Mr. Paolo Cavicchio and Ms. Maria Rodeano for providing material for histological analysis and to Ms. Francesca Bandoli for helping in sample collection. We would like to thank Dr. Jo Setchell for constructive comments on this paper.

\section{References}

Alaux C, Maisonnasse A, Le Conte Y (2010). Pheromones in a superorganism: from gene to social regulation. Vitamins and Hormones 83: 401-423.

Albone ES, Shirley SG (1984). Mammalian Semiochemistry. New York, Wiley \& Sons.

Chen WC, Zouboulis CC (2009). Hormones and the pilosebaceous unit. Dermatoendocrinology 1: 81-86. Epple G, Alveario MC, Golob NF, Smith AB III (1980). Stability and attractiveness related to age of scent marks of saddle back tamarins (Saguinus fuscicollis). Journal of Chemical Ecology 6: 735-748.

French JA, Cleveland J (1984). Scent-marking in the tamarin, Saguinus oedipus: sex differences and ontogeny. Animal Behaviour 32: 615-623.

French JA, Snowdon CT (1981). Sexual dimorphism in responses to unfamiliar intruders in the tamarin, Saguinus oedipus. Animal Behaviour 29: 822-829.

Mazzi V (1977). Manuale di tecniche istologiche e istochimiche. Padova, Piccin.

Moraes IA, De Carvalho MCA, De Azevedo Valle H, Pessoa VP, Ferreira AMP, Pissinatti A (2006). Histology of the sternal and suprapubic skin areas in lion tamarins (Leontopithecus sp. CallitrichidaePrimates). American Journal of Primatology 68: 1120-1126. 
Ozaki N, Suzuki M, Ohtaishi N (2004). Histological variations in myoepithelial cells and arrectores pilorum muscles among caudal, metatarsal and preorbital glands in Hokkaido sika deer (Cervus nippon yesoensis Heude, 1884). Journal of Veterinary Medical Science 66: 283-285.

Perkins EM (1969). The skin of primates. XL. The skin of the cotton-top pinche, Saguinus (Oedipomidas) oedipus. American Journal of Physical Anthropology 30: 13-27.

Quagliata S, Malentacchi C, Delfino C, Brunasso AMG, Delfino G (2006). Adaptative evolution of secretory cell lines in vertebrate skin. Caryologia 59: 187-206.

Scordato ES, Drea CM (2007). Scents and sensibility: information content of olfactory signals in the ringtailed lemur, Lemur catta. Animal Behaviour 73: 301-314.

Setchell JM, Vaglio S, Abbott KM, Moggi-Cecchi J, Boscaro F, Pieraccini G, Knapp LA (2011). Odour signals major histocompatibility complex genotype in an Old World monkey. Proceedings of the Royal Society B 278: 274-280.

Setchell JM, Vaglio S, Moggi-Cecchi J, Boscaro F, Calamai L, Knapp LA (2010). Chemical composition of scent-gland secretions in an Old World monkey (Mandrillus sphinx): influence of sex, male status, and individual identity. Chemical Senses 35: 205-220.

Stoeckelhuber M, Sliwa A, Welsch U (2000). Histo-physiology of the scent-marking glands of the penile pad, anal pouch, and the forefoot in the aardwolf (Proteles cristatus). Anatomical Record 259: 312-326.

Welsch U, van Dyk G, Moss D, Feuerhake F (1998). Cutaneous glands of male and female impalas (Aepyceros melampus): seasonal activity changes and secretory mechanism. Cell and Tissue Research 292: 377-394. 